\title{
Relative permeability prediction considering complex pore geometry and wetting characteristics in carbonate reservoirs
}

\section{Sunlee Han, Daewoong Kwak and Youngsoo Lee*}

Department of Mineral Resources and Energy Engineering, Chonbuk National University, 567 Baekje-daero, Deokjin-Gu, Jeonju-si, Jeollabuk-do 561-756, South Korea

Email: slhan@jbnu.ac.kr

Email: dayozzang77@jbnu.ac.kr

Email: youngsoo.lee@jbnu.ac.kr

\section{Youngho Jang}

Petroleum and Marine Research Division,

Korea Institute of Geoscience and Mineral Resources,

124 Gwahak-ro, Yuseong-gu,

Daejeon 34132, Korea

Email: yhjang@kigam.re.kr

\section{Wonmo Sung*}

Department of Natural Resources and Environmental Engineering,

Hanyang University,

222 Wangsimni-ro, Seongdong-gu,

Seoul 133-791, South Korea

Email: siro83@hanyang.ac.kr

Email: wmsung@hanyang.ac.kr

*Corresponding authors

\begin{abstract}
The measurement of relative permeability in carbonate rocks is very uncertain because of the complex pore system. Several equations for the relative permeability have been previously developed. However, most equations assume a single pore system that cannot be applied accurately. This study presents a relative permeability estimation method that considers capillary pressure, contact angle, pore size distribution, and residual oil saturation with respect to the heterogeneous pore network. As results, the capillary pressure was observed to have different tendencies for macropore and micropore. Also, the pore size distribution index of the macropore system was greater than the micropore system. For the macropore, water has a higher relative permeability than oil; a small reduction in oil saturation can easily flow water. In contrast, the micropore tends to follow the conventional relative permeability curve. Therefore, stable water displacement was not assured, leading to an early breakthrough for the heterogeneous carbonate rocks. [Received: July 11, 2016; Accepted: January 2, 2017]
\end{abstract}


Keywords: heterogeneous carbonate reservoir; relative permeability; pore system; capillary pressure; Brooks-Corey equation; two-phase flow.

Reference to this paper should be made as follows: Han, S., Kwak, D., Lee, Y., Jang, Y. and Sung, W. (2017) 'Relative permeability prediction considering complex pore geometry and wetting characteristics in carbonate reservoirs', Int. J. Oil, Gas and Coal Technology, Vol. 15, No. 4, pp.331-346.

Biographical notes: Sunlee Han received her BS degree in 2015 from Chonbuk National University. Currently, she is an MS student in Petroleum Engineering Lab. of Chonbuk National University. She is studying about fluid flow in complex pore network, $\mathrm{CO}_{2} \mathrm{EOR}$ and reservoir simulation.

Daewoong Kwak received his BS degree in Department of Mineral Resources and Energy Engineering from Chonbuk National University. He is an MS student at Chonbuk National University. His current research interest includes $\mathrm{CO}_{2}$ EOR and relative permeability in complex pore network.

Youngsoo Lee received his $\mathrm{PhD}$ in Petroleum and Natural Gas Engineering from Hanyang University in 2009. He is a Professor of Department of Mineral Resources and Energy Engineering at Chonbuk National University. His current research interests include geofluid flow analysis by using reservoir simulation and unconventional resources development (shale gas, tight gas, CBM).

Youngho Jang received his $\mathrm{PhD}$ in Petroleum and Natural Gas Engineering from Hanyang University. $\mathrm{He}$ is a researcher of Petroleum and Marine Research Division at Korea Institute of Geoscience and Mineral Resources. His research interests include nodal analysis, carbon sequestration, hydraulic fracturing and reservoir simulation.

Wonmo Sung received his $\mathrm{PhD}$ in Petroleum Engineering from Pennsylvania State University, USA. He is a Professor of the Department of Natural Resources and Environmental Engineering at Hanyang University. His current research interest includes polymer flooding, carbonate reservoir, carbon sequestration and fluid flow in unconventional reservoir.

This paper is a revised and expanded version of a paper entitled 'Relative permeability prediction considering complex pore geometry and wetting characteristics in carbonate reservoirs', presented at 2nd International Conference and Expo on Oil and Gas, Rome, 27-28 October 2016.

\section{Introduction}

In carbonate rocks, secondary pores such as dissolved pores, vugs, cavities, and fractures are well developed. Thus, carbonate rocks have heterogeneous pore systems caused by carbonate diagenesis. Most carbonate reservoirs have oil-wet characteristics, affecting not only fluid flow in the heterogeneous pore system but also oil production. In carbonate reservoirs, relative permeability measurement is extremely difficult because of the heterogeneous pore networks and wettability characteristics. In addition, relative permeability measurement is time consuming and has exhibited significant variation across experimental results, even for samples taken from the same formation. A number 
of correlations have been previously developed to explain multiphase flow in porous mediums; however, they cannot accurately predict the relative permeability of carbonates because they generally assume a homogeneous pore system and single pore size. In this study, we measured the pore size distribution, capillary pressure, and contact angle in a heterogeneous carbonate rock with wide-ranging pore sizes. Relative permeability curves were generated for the micropore and macropore using capillary pressure data, respectively. In carbonate rocks, there are not experimental identifications of the capillary pressure considering both micropore and macropore simultaneously. This study notably observed relative permeability characteristics by measuring capillary pressure for the different pore sizes. The results from this study can be used to explain the complex flow behaviour in heterogeneous carbonate reservoirs.

\section{Background}

\subsection{Carbonate reservoir characteristics}

More than $60 \%$ of the world's oil reservoirs are made of carbonate rock, and more than $80 \%$ of the carbonate rock has oil-wet characteristics. Carbonate rock that does not make contact with oil tends to have water-wet characteristics. Rao (1996) purported that the wettability of carbonate rock is changed to oil-wet by the chemical reaction between the rock surface and the oil surface activation components; when oil pressure exceeds critical capillary pressure, the thin layer of connate water on the rock surface is destroyed. Hamouda and Karam (2006) considered the altered wettability of carbonate rock. Due to the loss of hydrogen ion, the carboxyl group of oil presents a negative electric charge and adheres to the positively charged rock surface. For the oil-wet rock, water flows more easily than oil through the pore networks that oil recovery can be less than $10 \%$ (Montaron, 2005). Water flooding simulations show that only limited amounts of oil can be recovered from oil-wet layers because the water tends to flow primarily through the water-wet layers in carbonate reservoirs (Schlumberger, 2007). The heterogeneous pore system and difficulties in fluid flow prediction require an enhanced understanding of fundamental carbonate reservoir characteristics.

\subsection{Pore size distribution}

Heterogeneous pore systems comprising dissolved pores, vugs, cavities, and fractures significantly affect permeability. For this reason, it is important to analyse reservoir properties with respect to pore size. Hwang (2010) proposed a more precise prediction model for drainage capillary pressure, which considers micropore and macropore size effects in heterogeneous carbonate rock. He confirmed that this approach was more reliable than existing capillary pressure models that assume a homogeneous pore system. Accordingly, pore size analysis is essential for heterogeneous carbonate reservoirs. Several pore size measurement methods exist, including the gas adsorption, mercury injection, micro-tomography, and electron microscopy methods. Among these methods, gas adsorption measures pore sizes of $0.3-300 \mathrm{~nm}$, and mercury injection measures pore sizes of $3 \mathrm{~nm}-1 \mathrm{~mm}$ (Alonso and Alberto, 2005). Vugs and channel pores larger than a few millimetres are generally problematic to characterise because they may be larger than the plug sample. The mercury porosimeter is the only method suitable for plug-sample or 
smaller sizes (Hulea and Nicholls, 2012). Therefore, use of the mercury porosimeter was deemed appropriate for the carbonate rock analysis with wide-ranging pore sizes.

\subsection{Capillary pressure}

For a formation with a complex pore system, capillary pressure should be determined by special core experiment. The mercury injection method is widely used because it can measure not only pore size but also capillary pressure. A non-wetting fluid such as mercury is injected into the sample, penetrating smaller pores as the injection pressure increases. The resulting measurements are expressed as a function of the cumulative penetration volume of injected mercury depending on pressure or pore size. As recommended by Purcell (1949) and Schowalter (1979), the relationship between the applied pressure and the pore throat radius into which the mercury will penetrate is given by the modified Washburn equation as follows (Daniel and Kaldi, 2010):

$$
r=\frac{2 I F T \cos \theta}{P_{c}}
$$

where $r$ is the pore throat radius, IFT is the interfacial tension of mercury and air, $\theta$ is the contact angle between mercury and the pore wall, and $P_{c}$ is the applied capillary pressure.

Assuming that the pore throat sizes in the laboratory were identical to those in the reservoir, the capillary pressure for the reservoir condition $\left(P_{c, r e s}\right)$ can be calculated as follows(Torsæter and Abtahi, 2003):

$$
P_{c, r e s}=\frac{I F T_{\text {res }} \cos \theta_{\text {res }}}{I F T_{\text {lab }} \cos \theta_{\text {lab }}} P_{c, l a b}
$$

where $\theta$ is the contact angle for reservoir (res) and laboratory (lab) conditions, respectively, and $P_{c, l a b}$ is the capillary pressure for the laboratory condition. Here, interfacial tension and contact angle between immiscible fluids and the reservoir rock need to be converted from laboratory conditions to reservoir conditions. However, it is difficult to determine the exact value of the contact angle for fluids in a porous matrix (Dandekar, 2013). Thus, the contact angles are often neglected and equation (2) is reformulated as follows:

$$
P_{c, r e s}=\frac{I F T_{\text {res }}}{I F T_{\text {lab }}} P_{c, l a b}
$$

The $I F T_{\text {res }}$ between mercury and air changes with pore size and the $I F T_{\text {lab }}$ converges to $475.5 \mathrm{mN} / \mathrm{m}$ for pore sizes of 30-500 $\mu \mathrm{m}$ (Wang et al., 2016). Lombard et al. (2004) observed that all capillary pressure curves converge to a single capillary pressure curve for homogeneous samples, while the $P_{c}$ data remains scattered for heterogeneous samples. Kim et al. (2011) presented superimposed type of capillary pressure model according to pore size in heterogeneous carbonate rock. Hulea and Nicholls (2012) developed a saturation model for a capillary pressure curve according to pore size in heterogeneous carbonate rock. Also, Joekar-Niasar et al. (2008) analysed the relationships among capillary pressure, interfacial area, saturation, and relative permeability in porous media. Figure 1 shows capillary pressure curves for homogeneous and heterogeneous carbonate rock, respectively. 
Figure 1 Previously derived capillary pressure curves, (a) for homogeneous carbonate rock (b) for heterogeneous carbonate rock (see online version for colours)

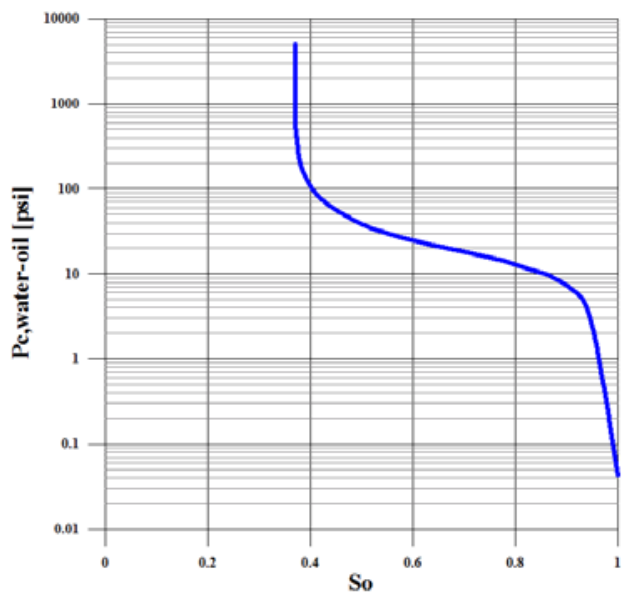

(a)

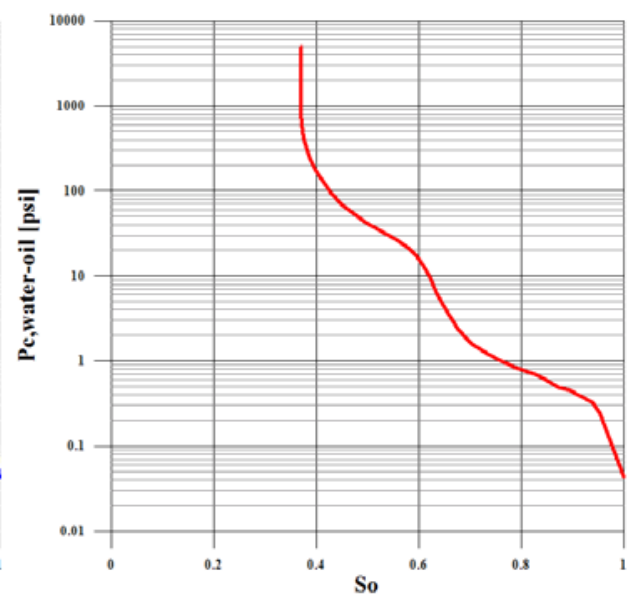

(b)

\subsection{Relative permeability}

Determination of relative permeability is essential for reservoir productivity and multiphase flow analyses. However, this method is expensive, complex and time consuming. Roghanian et al. (2012) used correlations between residual and initial saturation to predict relative endpoint permeability and found that the results were not substantially different from experimental results. Other proposed correlations use effective phase saturation as a correlating parameter. The effective phase saturation is defined as follows (Ahmed, 2010):

$$
\begin{aligned}
& S_{o}^{*}=\frac{S_{o}}{1-S_{w c}} \\
& S_{w}^{*}=\frac{S_{w}-S_{w c}}{1-S_{w c}} \\
& S_{g}^{*}=\frac{S_{g}}{1-S_{w c}}
\end{aligned}
$$

where $S_{o}^{*}, S_{w}^{*}$ and $S_{g}^{*}$ are the effective oil, water, and gas saturation, respectively; $S_{o}$, $S_{w}$, and $S_{g}$ are the oil, water, and gas saturation, respectively; and $S_{w c}$ is the irreducible water saturation. Several two-phase relative permeability correlations have been previously developed, including the Wyllie and Gardner, Torcaso and Wyllie, Pirson and Brooks-Corey equations. The Wyllie and Gardner equation determines relative permeability according to formation type for unconsolidated sand, cemented sandstone, and oolitic limestone (Wyllie and Gardner, 1958; Honarpour et al., 1986). The Torcaso and Wyllie equation determines relative permeability using the relative permeability for gas and the effective phase saturation; relative permeability measurements for gas are more easily captured than measurements for oil in a gas-oil system (Torcaso and Wyllie, 
1958). The Pirson equation determines the wetting and non-wetting phases for water-wet rocks relative to permeability for both imbibition and drainage processes (Pirson, 1958).

The Brooks-Corey equation is most widely applied because it depicts the relationship between relative permeability and capillary pressure. Lian et al. (2015) developed a saturation model for a carbonate reservoir using the Brooks-Corey equation and recommended its use for carbonate reservoirs because it was specifically developed to describe capillary pressure curves, and effectively represents both long and short transition zones. Brooks-Corey equation modified Corey's original drainage capillary pressure-saturation relationship and combined this modified equation with Burdines's equation to develop the following expression that predicts drainage relative permeability for any pore size distribution (Corey, 1954):

$$
S_{w}^{*}=\left(\frac{P_{d}}{P_{c}}\right)^{\lambda}
$$

where $P_{d}$ is the displacement pressure, and $\lambda$ is a factor that represents pore size variation in the medium. The parameters $P_{c}$ and $\lambda$ are characteristic of the medium and $P_{d}$ approximates the minimum drainage capillary pressure at which a continuous non-wetting phase exists in a porous medium. To determine $\lambda$ and $P_{d}$, a straight trend line is produced for $\left(S_{o}-S_{o i}\right) /\left(1-S_{o i}\right)$ versus the capillary pressure curve in a log-log scale. Using this relationship, the two-phase relative permeabilities can be determined as follows (Brooks and Corey, 1964):

$$
\begin{aligned}
& k_{r w}=\left(S_{w}^{*}\right)^{\frac{2+3 \lambda}{\lambda}} \\
& k_{r n w}=\left(1-S_{w}^{*}\right)^{2}\left[1-\left(S_{w}^{*}\right)^{\frac{2+\lambda}{\lambda}}\right]
\end{aligned}
$$

where $k_{r w}$ and $k_{r n w}$ are the relative permeabilities for the wetting and non-wetting phases, respectively.

Theoretically, $\lambda$ may assume any value greater than zero; larger values reflect a relatively uniform medium while smaller values reflect a medium with greater pore size variation (Honarpour et al., 1986). Belayneh et al. (2006) successfully simulated water injection using the Brooks-Corey relative permeability equation in a carbonate reservoir. Byrnes and Bhattacharya (2006) developed a capillary pressure model for a heterogeneous carbonate reservoir using a modified Brooks-Corey equation and studied the effects of initial/residual oil saturation and relative permeability on recovery. Hulea and Nicholls (2012) fitted a capillary pressure curve to a saturation model for heterogeneous carbonate rock using the Brooks-Corey equation.

In general, both micropore and macropore are developed in complex carbonate rocks. The previously developed empirical correlations described here assume a single pore system, resulting in a high degree of uncertainty in relative permeability estimation. Because capillary pressure is different according to the pore structure, precise relative permeability prediction requires consideration of pore size effects. In addition, capillary pressure and wetting characteristics must be concurrently considered to determine oil-water two-phase flow behaviour in highly heterogeneous carbonate reservoirs. The results of this study help to explain two-phase relative permeability in complex carbonate pore networks. 


\section{Result}

\subsection{Contact angle}

To develop a capillary pressure relationship, the contact angle and interfacial tension between the reservoir fluids and rock must be measured. However, these measurements are too difficult to obtain, particularly for the crude oil sample. In this study, we used decane, which has an already established interfacial tension correlation with water. And, limestone core which is made for the experimented purpose is used.

When measuring the contact angle which is used as an indicator of wettability, one small drop of wetting fluid is placed on a rock surface that is already fully saturated with non-wetting fluid. Contact angle measurement (drop shape analyser - DSA100) was used to measure the contact angle. The particularly uniform LED illumination and the quality lens have high precision in order to accurately measure the contact angle or surface tension. If the contact angle of the oil droplet is greater than $90^{\circ}$, solids exhibit oil-wet characteristics in solid-oil-water systems (He et al., 2015).

Figure 2 Contact angles in carbonate rock, (a) with no oil contact (b) after 72 hours oil saturation (see online version for colours)

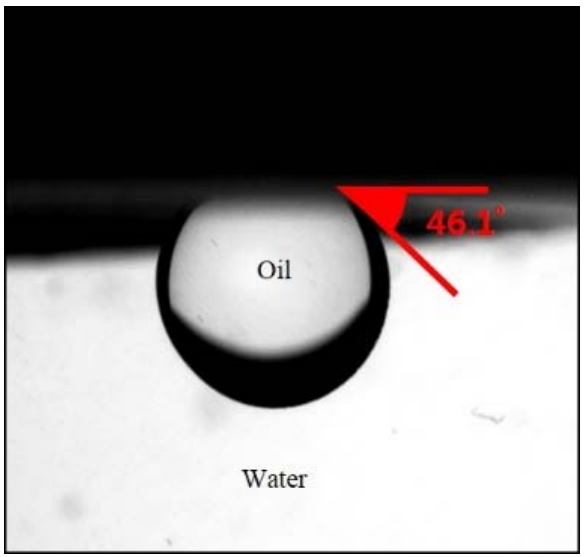

(a)

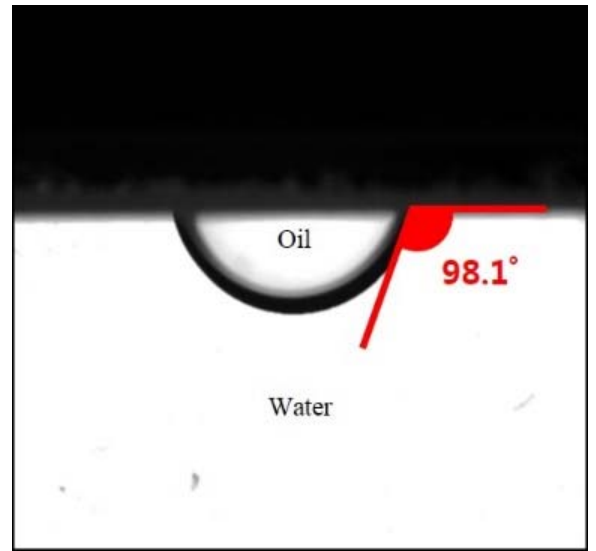

(b)

As shown in Figure 2(a), a carbonate rock surface that does not contact oil has hydrophilic characteristics. However, a carbonate oil reservoir has contacted oil at high pressures and temperatures over thousands of years. Over this extended period, the wettability of the carbonate rock changes because of increased ionic bonds between the oil carboxyl and the carbonate rock surface. Water wetness reportedly decreases as contact time of oil increases (Zhou et al., 2000). The change of oil production rate was observed to be extremely low after aging the cores at reservoir pressure for 24 hours compared to 72 hours (Zhou et al., 1995). It means that over than 24 hours of aging time has no effect to wettability change. In this study, in order to make initial reservoir condition, carbonate core was saturated with decane for 72 hours and contact angle was measured as $98.1^{\circ}$. It indicates that the core used in this study shows oil-wet characteristics [Figure 2(b)]. 
Yang et al. (2007) reported that the contact angle increased more than $20^{\circ}$ in an oil-wet sample as pressure increased. Ameri et al. (2013) confirmed the considerable dependence of the contact angle on pressure at the oil-wet surface. Accordingly, the wettability of reservoir rock may change to oil-wet in the reservoir condition.

\subsection{End-point measurement}

In order to prevent pore size change, carbonate sample was treated by pre-flushing of water at reservoir condition. Reservoir condition was assumed to be 1,100 psi and 293.15 K, respectively. After the carbonate core was fully saturated with decane using syringe pump (Figure 3), residual oil saturation and maximum water relative permeability were obtained by injecting water. Finally, irreducible water saturation and maximum oil relative permeability were measured by re-injecting oil until water was no longer flowed. Table 1 summarises these results.

Figure 3 Experimental setup for special core analysis (see online version for colours)

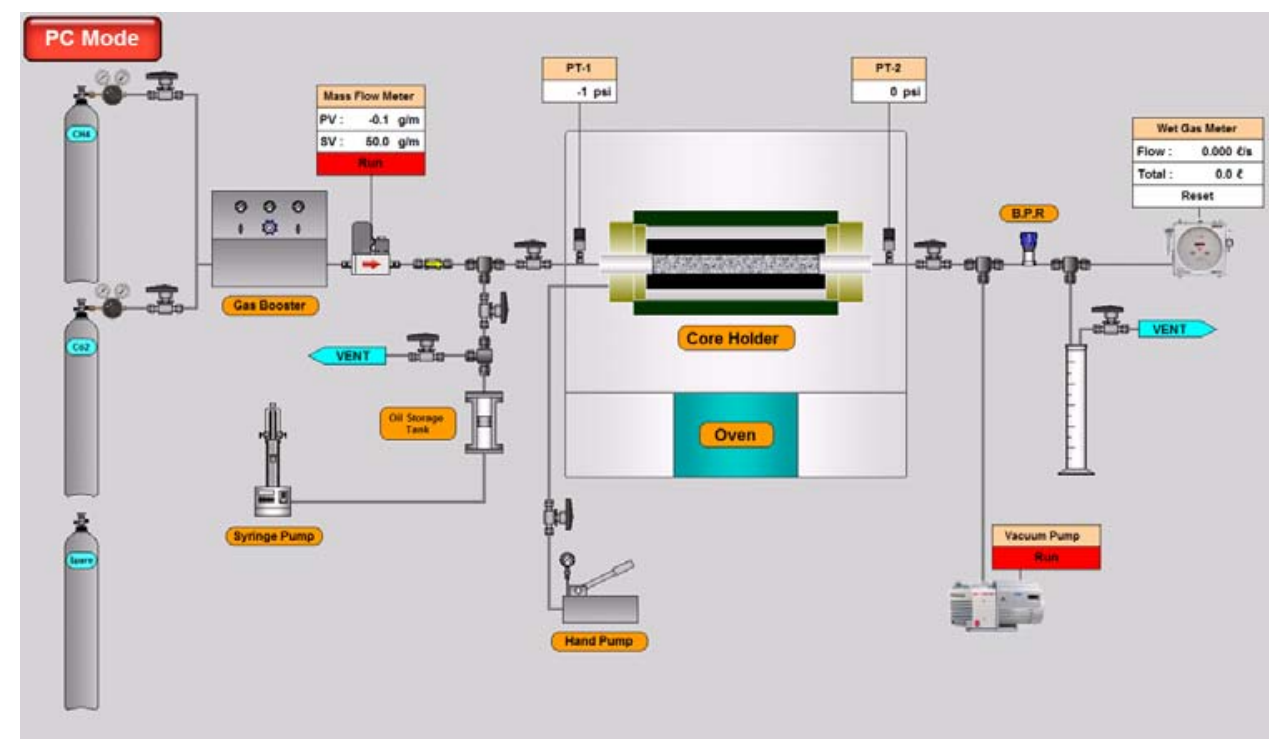

Table 1 Endpoint results from special core analysis

\begin{tabular}{lcc}
\hline & Oil & Water \\
\hline End point saturation & 0.37 & 0.14 \\
Maximum relative permeability & 0.13 & 0.32 \\
\hline
\end{tabular}

\subsection{Mercury porosimeter}

\subsubsection{Pore size measurement}

To estimate the relative permeability, endpoints, capillary pressure, and pore size distribution must be determined. Mercury porosimetry characterises porosity by applying various levels of pressure to a sample immersed in mercury. The pressure required for 
mercury to penetrate the sample's pores is inversely proportional to the size of the pores; the pore size distribution can be subsequently determined (Kantzas et al., 2014). In this study, Autopore III Porosimeter 9500 was used to measure the pore size distribution. Following transfer to a high-pressure system, the sample is surrounded by hydraulic fluid, and isostatic pressures of up to 60,000 psi are applied (Giesche, 2006; Daniel and Kaldi, 2010). Minimum pore sizes of $3.5 \mathrm{~nm}$ can be determined using a mercury porosimeter.

Table 2 Pore system classification by pore size

\begin{tabular}{lcc}
\hline Micropore & Mesopore & Macropore \\
\hline$<1 \mu \mathrm{m}$ micro-pore throat & $1-3 \mu \mathrm{m}$ micro-pore throat \\
diameters & diameters & $3 \mu \mathrm{m}>$ micro-pore throat \\
diameters
\end{tabular}

Source: Bennion and Bachu (2006)

Figure 4 Mercury injection volume relative to pore size, (a) pore size distribution (b) cumulative volume of injected mercury (see online version for colours)

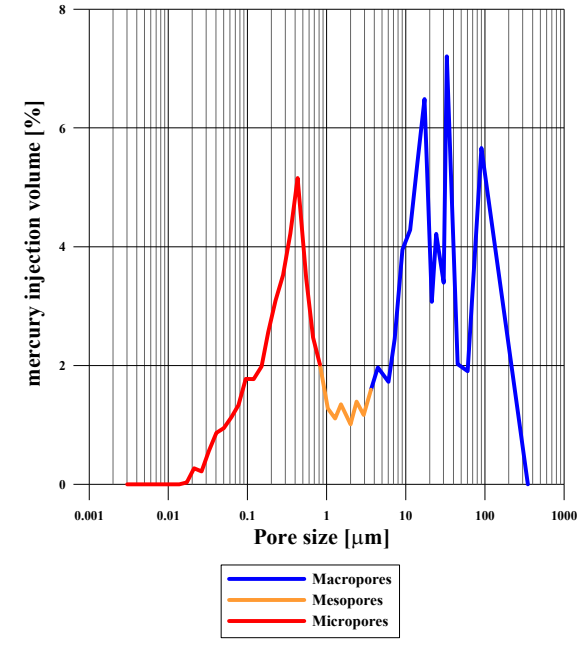

(a)

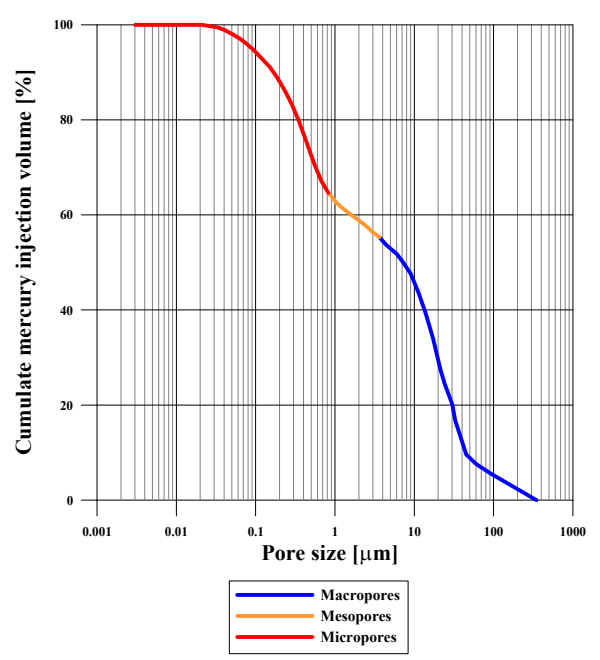

(b)

Following mercury porosimetry analysis, the pore system is classified into three groups:

1 micropore

2 mesopore

3 macropore (Bennion and Bachu, 2006).

Table 2 lists the respective pore sizes in each classification, relative to a pore throat diameter. Figure 4(b) shows the experimental result of cumulative volume of injected mercury relative to pore size. If the pore size distribution has a normal distribution, the cumulative volume of injected mercury has a single bend shape. However, if the pore size is heterogeneous [see Figure 4(a)], the cumulative volume curve has a double bend shape. The carbonate sample pore system primarily comprised micropore and macropore. The amount of injected mercury varied with respect to the pore structure and injection pressure; smaller pore sizes require higher pressures for mercury penetration. In this 
study, the capillary pressure and relative permeability of the carbonate with different pore sizes were analysed.

Figure 5 Capillary pressure curves derived in this study; general form for primary drainage (see online version for colours)

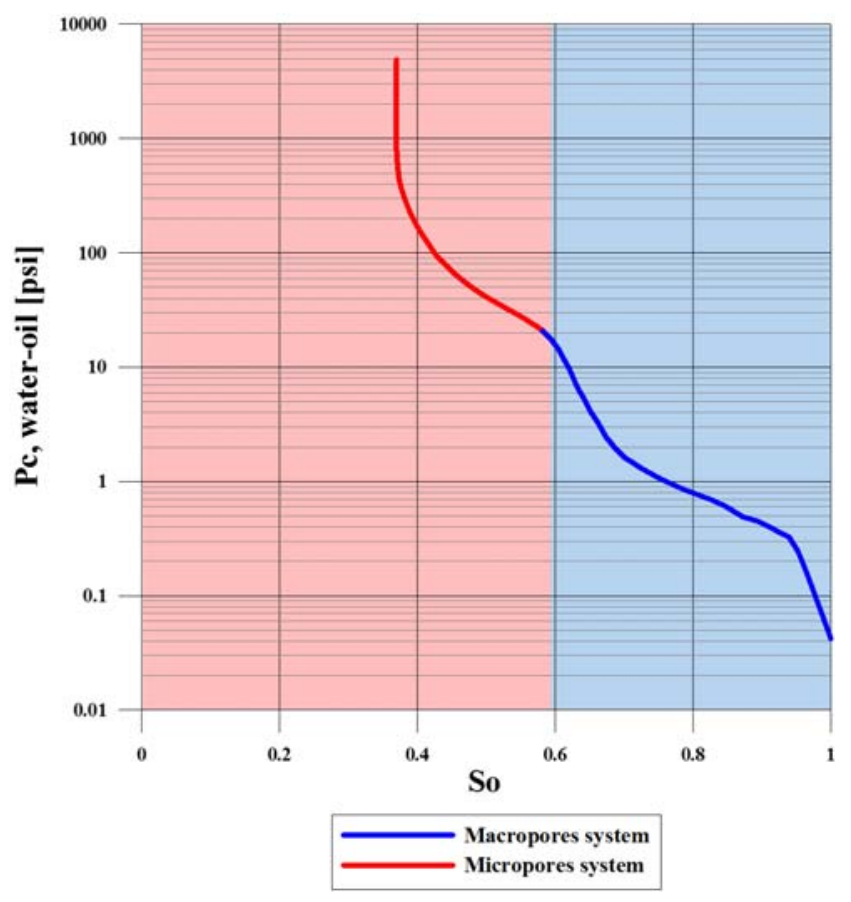

\subsubsection{Capillary pressure measurement}

Capillary pressure measurements captured under laboratory conditions using a mercury-air combination were converted to water-oil system that exists under reservoir condition (Brown, 1951). The pressure and temperature for the reservoir condition was assumed to be $1,100 \mathrm{psi}$ and $293.15 \mathrm{~K}$, respectively. To establish capillary pressure with wetting phase saturation for this reservoir condition, interfacial tension between decane and water was required. Jennings (1967) measured the interfacial tension for decane-water at pressures of 14.7-12,007 psi and temperatures of 298.15-449.15 K. Over this range of pressures and temperatures, the interfacial tension for a decane-water system can be expressed as follows:

$$
I F T=50.066+1.8540 \times 10^{-4} \times P-0.12050(T-298.15)
$$

where $I F T$ is the interfacial tension $[\mathrm{mN} / \mathrm{m}], P$ is the pressure [psi] and $T$ is the temperature $[\mathrm{K}]$.

A simple normalisation equation without any weighting factors or higher-order functions was used to combine mercury injection capillary pressure curves and residual oil saturation as follows (Nemes, 2016): 


$$
S_{w_{n}}=S_{w_{n} \min }+\frac{S_{w}-S_{w_{\min }}}{S_{w_{\max }}-S_{w_{\text {min }}}} \times\left(S_{w_{n \text { max }}}-S_{w_{n \text { min }}}\right)
$$

where $S_{w}$ is the wetting fluid saturation of minimum (min), maximum (max) and/or in a normalised state $(n)$.

In this study, mesopore were considered as macropore because of their small proportion in the sample. Capillary pressures with normalised saturation were calculated using equations (3) and (11) that pore systems were divided into macropore and micropore systems, respectively. Figure 5 depicts the superimposed capillary pressure curves. Fluid flow occurred stepwise from the macropore to the micropore. Fluids did not concurrently flow in the micropore because of the smaller displacement pressures in the macropore. Because the capillary pressure changes with the carbonate rock pore system, the effect of the pore system must be considered when explaining relative permeability in a heterogeneous carbonate.

\subsection{Relative permeability prediction}

To predict the relative permeability using the Brooks-Corey equation, $\left(S_{o}-S_{o i}\right) /\left(1-S_{o i}\right)$ versus the capillary pressure curve in a log-log scale was drawn, and is depicted in Figure 6. As shown in Figure 6(a), $\lambda$ and $P_{d}$ cannot be determined in a heterogeneous rock because the relationship between $\left(S_{o}-S_{o i}\right) /\left(1-S_{o i}\right)$ and capillary pressure is nonlinear. However, as shown in Figure 6(b), a strong linear trend was observed for each pore system.

Figure $6 \quad\left(S_{o}-S_{o i}\right) /\left(1-S_{o i}\right)$ versus capillary pressure for the (a) combined pore system and (b) individual pore systems (see online version for colours)

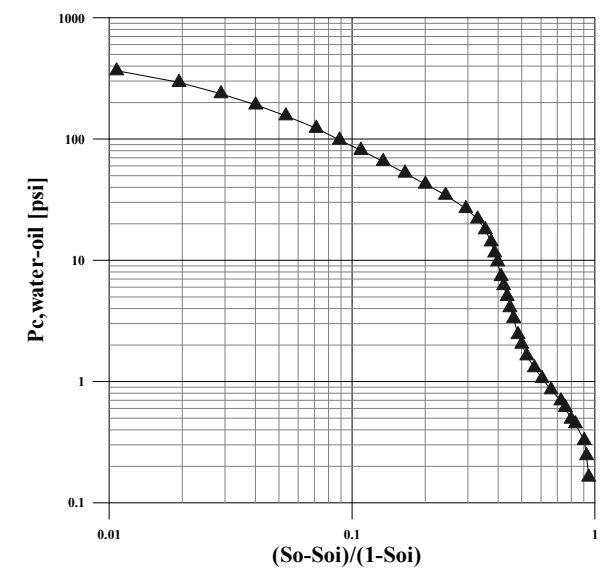

(a)

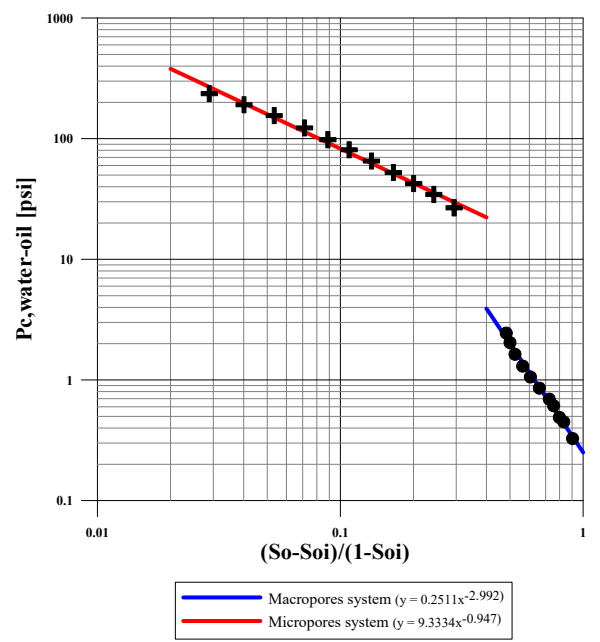

(b)

If a pore system is homogeneous, $P_{d}$ has a uniform value. However, our results clearly indicate large variations in $P_{d}$, suggesting a highly heterogeneous pore system in the sample. Additionally, the micropore system, with a smaller $\lambda$, had a wider range of pore sizes than the macropore system. Figure 7 depicts the capillary pressure curves with 
normalised saturation that were newly calculated using $\lambda$ and $P_{d}$ by Brooks-Corey equation for the each pore system. $P_{d}$ and residual oil saturation $\left(S_{o r}\right)$ was calculated as $9.3334 \mathrm{psi}$ and 0.37 for the micropore system. In contrast, $P_{d}$ and $S_{o r}$ was calculated as $0.2511 \mathrm{psi}$ and 0.5 for the macropore system. It means that the resistance of micropores to water intrusion is much higher than the case of macropores.

Figure 7 Capillary pressure curves derived according to the pore system (see online version for colours)

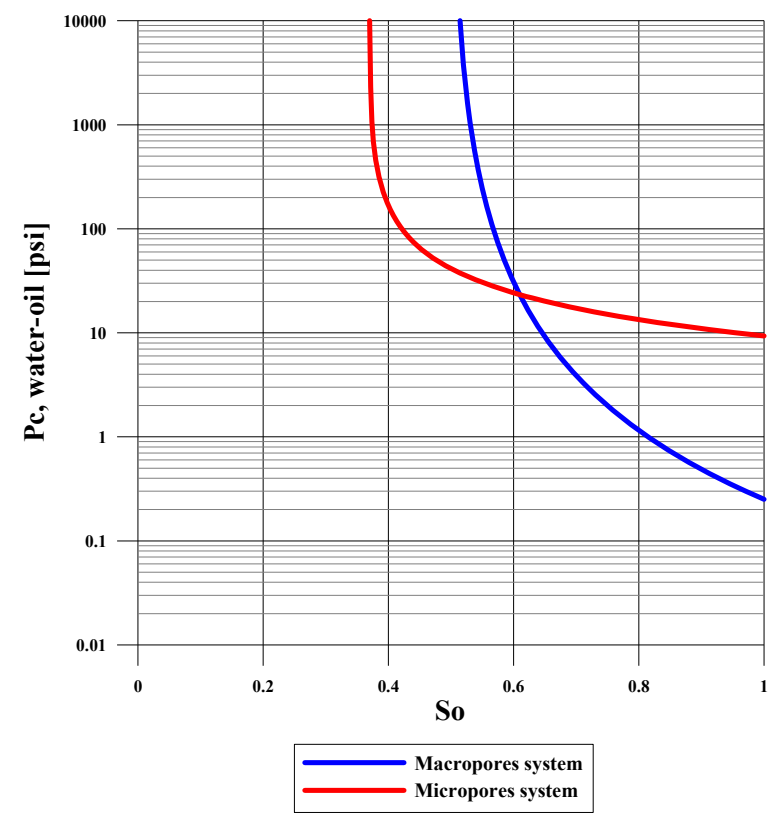

Next, the relative permeability was calculated using the Brooks-Corey equation for the pore system shown in Figure 8. As shown in Figure 8(a), a small reduction in oil saturation drastically reduced the relative permeability of oil. For the macropore system, the relative permeability of oil is almost zero below an oil saturation of 0.9. Under these conditions, only water that occupies the macropore flows. As shown in the water relative permeability curve, water begins to flow at relatively high oil saturation and water penetrates more easily in the macropores because of a smaller $P_{d}$. Thus, in oil-wet condition for the macropore system, oil is stationary and water flows easily through the central pore network at high oil saturations. In practice, when water flooding is applied to a carbonate reservoir, water cannot effectively displace oil, and early breakthroughs occur due to the preferential flow of water in the macropore system.

In contrast, as shown in Figure 8(b), the intersection of relative permeabilities for water and oil in the micropore system occurred at an oil saturation of 0.7 indicating an oil-wet condition. This means that water flows better as oil saturation decreases, and substantial amount of oil is trapped due to the high residual oil saturation. However, greater pressures are required for the water to penetrate the pore; oil preferentially occupies the smaller pore spaces where capillary forces are the greatest. Therefore, in practice, it is very difficult to enter the water into micropore in heterogeneous carbonate reservoir because most water flows through the macropore. 
Figure 8 Drainage relative permeability for the (a) macropore system and (b) micropore system (see online version for colours)

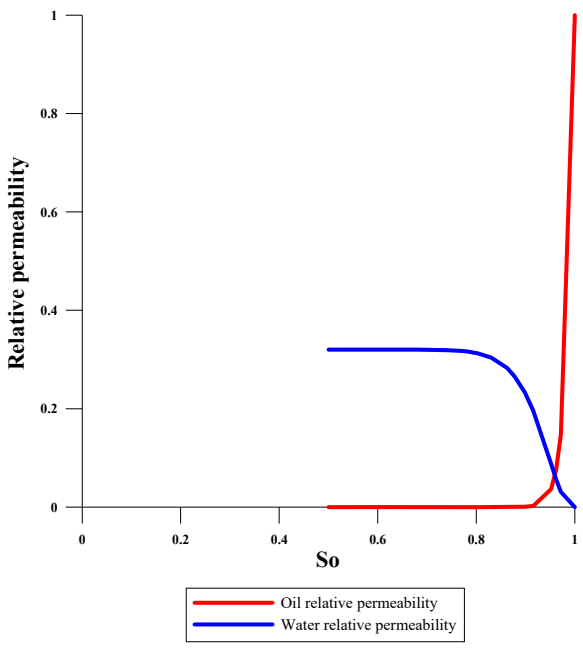

(a)

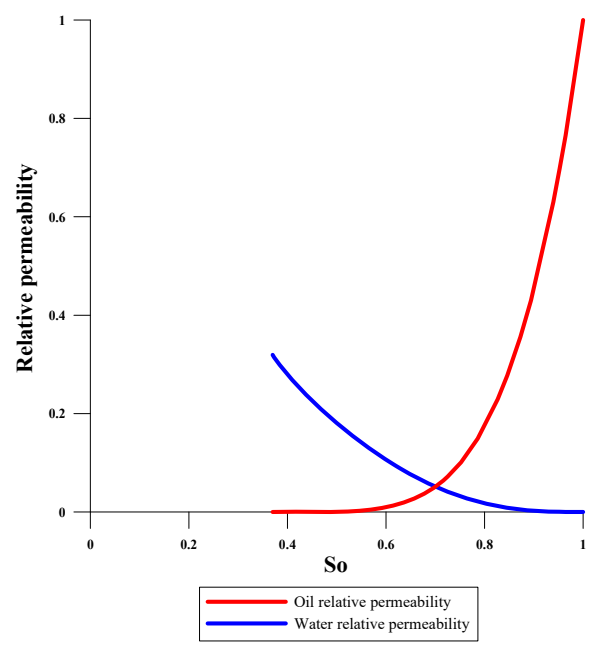

(b)

\section{Conclusions}

This study presents a relative permeability estimation method with respect to the heterogeneous pore network that considers the capillary pressure, contact angle, pore size distribution, and residual oil saturation. As a result, the pore size distribution index of the macropore system was greater than that of the micropore system, indicating a wider pore size range for the micropore system. The results of this study regarding capillary pressure based on $\lambda$ and $P_{d}$ suggest that the relative permeability of the carbonate sample was likely affected by pore size. Therefore, oil and water were observed to flow differently through micropore and macropore, according to the oil saturation and stable water displacement was not assured, leading to an early breakthrough. In conclusion, the preferential flow of water through the macropore and the resistance of micropore to water penetration are the primary reasons for low oil recovery in carbonate reservoirs. Thus, improved water penetration for the micropore system which can be accomplished by increasing water injection pressure or water-oil capillary pressure is the key to enhanced oil recovery. However, for the micropore system, increasing capillary pressure is difficult to achieve because water flows through the highly permeable macropore. For this reason, capillary pressure cannot be maintained above the pressure level required for water penetration, resulting in a very small amount of recoverable oil in the micropore system. 


\section{Acknowledgements}

This research was supported by Basic Science Research Program through the National Research Foundation of Korea (NRF) funded by the Ministry of Education (No.2014R1A1A1036529) and the Energy Efficiency and Resources Core Technology Program of the Korea Institute of Energy Technology Evaluation and Planning (KETEP), granted financial resource from the Ministry of Trade, Industry \& Energy, Republic of Korea (No. 20132510100060).

\section{References}

Ahmed, T. (2010) Reservoir Engineering Handbook, Gulf Professional Publishing, USA.

Alonso, E. and Alberto, L. (2005) Advances in Understanding Engineered Clay Barriers, Balkema Pub, Leiden.

Ameri, A., Kaveh, N.S., Rudolph, E., Wolf, K.H., Farajzadeh, R. and Bruining, J.A. (2013) 'Investigation on interfacial interactions among crude oil-brine-sandstone rock- $\mathrm{CO}_{2}$ by contact angle measurements', Energy \& Fuels, Vol. 27, No. 2, pp.1015-1025.

Belayneh, M., Geiger, S. and Matthi, S.K. (2006) 'Numerical simulation of water injection into layered fractured carbonate reservoir analogs', AAPG Bulletin, Vol. 90, No. 10, pp.1473-1493.

Bennion, D.B. and Bachu, S. (2006) 'The impact of interfacial tension and pore size distribution/capillary pressure character on $\mathrm{CO}_{2}$ relative permeability at reservoir conditions in $\mathrm{CO}_{2}$-brine systems', SPE/DOE Symposium on Improved Oil Recovery.

Brooks, R.H. and Corey, A.T. (1964) 'Hydraulic properties of porous media and their relation to drainage design', Transactions of the ASAE, Vol. 7, No. 1, pp.26-28.

Brown, H.W. (1951) 'Capillary pressure investigations', Journal of Petroleum Technology, Vol. 3, No. 3, pp.67-74.

Byrnes, A.P. and Bhattacharya, S. (2006) 'Influence of initial and residual oil saturation and relative permeability on recovery from transition zone reservoirs in shallow-shelf carbonates', SPE/DOE Symposium on Improved Oil Recovery, Society of Petroleum Engineers.

Corey, A.T. (1954) 'The interrelation between gas and oil relative permeabilities', Producers Monthly, Vol. 19, No. 1, pp.38-41.

Dandekar, A.Y. (2013) Petroleum Reservoir Rock and Fluid Properties, CRC Press, Boca Raton.

Daniel, R.F. and Kaldi, J.G. (2010) Evaluating Mercury Injection Capillary Pressure (MICP) Data to Determine CO2 Column Height Retention of Caprocks, CO2CRC, Barton.

Giesche, H. (2006) 'Mercury porosimetry: a general (practical) overview', Particle \& Particle Systems Characterization, Vol. 23, No. 1, pp.9-19.

Hamouda, A.A. and Karam, A.R.G. (2006) 'Influence of temperature on wettability alteration of carbonate reservoirs', SPE/DOE Symposium on Improved Oil Recovery, Society of Petroleum Engineers.

He, L., Lin, F., Li, X., Sui, H. and Xu, Z. (2015) 'Interfacial sciences in unconventional petroleum production: from fundamentals to applications', Chemical Society Reviews, Vol. 44, No. 15, pp.5446-5494.

Honarpour, M.M., Koederitz, F. and Herbert, A. (1986) Relative Permeability of Petroleum Reservoirs, pp.16-41, CRC Press, Boca Raton.

Hulea, I.N. and Nicholls, C.A. (2012) 'Carbonate rock characterization and modeling: capillary pressure and permeability in multimodal rocks - a look beyond sample specific heterogeneity', AAPG Bulletin, Vol. 96, No. 9, pp.1627-1642.

Hwang, S.H. (2010) A Development of Capillary Pressure Model Considering Heterogeneous Pore Geometry in Carbonate Reservoir, Master's thesis, Hanyang University, Seoul, Korea. 
Jennings, H.Y. (1967) 'The effect of temperature and pressure on the interfacial tension of benzene-water and normal decane-water', Journal of Colloid and Interface Science, Vol. 24, No. 3, pp.323-329.

Joekar-Niasar, V., Hassanizadeh, S.M. and Leijnse, A. (2008) 'Insights into the relationships among capillary pressure, saturation, interfacial area and relative permeability using pore-network modeling', Transport in Porous Media, Vol. 74, No. 2, pp.201-219.

Kantzas, A., Bryan, J. and Taheri, S. (2014) Fundamentals of Fluid Flow in Porous Media [online] $\mathrm{http}: / /$ perminc.com/resources/fundamentals-of-fluid-flow-in-porous-media/ (accessed 19 May 2016).

Kim, K., Lee, Y., Hwang, S., Seo, J. and Sung, W. (2011) 'Improved capillary pressure model considering dual-pore geometry system in carbonate reservoirs', Journal of Petroleum Science and Engineering, Vol. 78, No. 3, pp.601-608.

Lian, P., Tan, X., Ma, C., Feng, R. and Gao, H. (2015) 'Saturation modeling in a carbonate reservoir using capillary pressure based saturation height function: a case study of the Svk reservoir in the Y Field', Journal of Petroleum Exploration and Production Technology, Vol. 6, No. 1, pp.73-84.

Lombard, J., Egermann, P., Lenormand, R., Bekri, S., Hajizadeh, M., Hafez, H., Modavi, A. and Kalam, M.Z. (2004) 'Heterogeneity study through representative capillary pressure measurements-Impact on reservoir simulation and field predictions', International Symposium of the Society of Core Analysts, Pau, France, Abu Dhabi, pp.5-9.

Montaron, B. (2005) 'Increasing oil recovery factors: a technical challenge key to future world energy supply', AFTP 2005, Paris.

Nemes, I. (2016) 'Revisiting the applications of drainage capillary pressure curves in water-wet hydrocarbon systems', Open Geosciences, Vol. 8, No. 7, pp.22-38.

Pirson, S.J. (1958) Oil Reservoir Engineering, McGraw-Hill, New York.

Purcell, W.R. (1949) 'Capillary pressures - their measurement using mercury and the calculation of permeability therefrom', Journal of Petroleum Technology, Vol. 1, No. 2, pp.39-48.

Rao, D.N. (1996) 'Wettability effects in thermal recovery operations', SPE/DOE Improved Oil Recovery Symposium, Vol. 2, No. 5, pp.420-430, Society of Petroleum Engineers.

Roghanian, R., Rasaei, M.R. and Haghighi, M. (2012) 'Prediction of key points of water-oil relative permeability curves using the linear regression technique', Petroleum Science and Technology, Vol. 30, No. 5, pp.518-533.

Schlumberger (2007) Carbonate Reservoirs [online] http://www.slb.com/ /media/Files/industry challenges/carbonates/brochures/cb_carbonate_reservoirs_07os003.pdf (accessed 21 April 2016).

Schowalter, T.T. (1979) 'Mechanics of secondary hydrocarbon migration and entrapment', AAPG Bulletin, Vol. 63, No. 5, pp.723-760.

Torcaso, M.A. and Wyllie, M. (1958) 'A comparison of calculated krg/kro ratios with a correlation of field data', Journal of Petroleum Technology, Vol. 10, No. 12, pp.57-58.

Torsæter, O. and Abtahi, M. (2003) Experimental Reservoir Engineering Laboratory Workbook, Department of Petroleum Engineering and Applied Geophysics, Norwegian University of Science and Technology (NTNU), Trondheim.

Wang, S., Javadpour, F. and Feng, Q. (2016) 'Confinement correction to mercury intrusion capillary pressure of shale nanopores', Scientific Reports, Vol. 6, No. 20160, pp.1-11.

Wyllie, M.R.J. and Gardner, G.H.F. (1958) 'The generalized Kozeny-Carmen equation its application to problems of multi-phase flow in porous media', World Oil, Vol. 146, No. 121, pp.210-227.

Yang, D., Gu, Y. and Tontiwachwuthikul, P. (2007) 'Wettability determination of the reservoir brine-reservoir rock system with dissolution of $\mathrm{CO}_{2}$ at high pressures and elevated temperatures', Energy \& Fuels, Vol. 22, No. 1, pp.504-509. 
Zhou, X.M., Torsaeter, O., Xie, X. and Morrow, N.R. (1995) 'The effect of crude-oil aging time and temperature on the rate of water imbibition and long-term recovery by imbibition', SPE Formation Evaluation, Vol. 10, No. 4, pp.259-266.

Zhou, X.M., Morrow, N.R. and Ma, S. (2000) 'Interrelationship of wettability, initial water saturation, aging time, and oil recovery by spontaneous imbibition and waterflooding', SPE Journal, Vol. 5, No. 2, pp.199-207. 